son with isoflurane. Anesthesiology 1993; 79: 444-53.

16 Matta BF, Heath KJ, Tipping K, Summors AC. Direct cerebral vasodilatory effects of sevoflurane and isoflurane. Anesthesiology 1999; 91: 677-80.

17 Sudikoff S, Banasiak K. Techniques for measuring cerebral blood flow in children. Curr Opin Pediatr 1998; 10: 291-8.

18 Bissonnette B, Benson LN. Closure of persistently patent arterial duct and its impact on cerebral circulatory haemodynamics in children. Can J Anaesth 1998; 45: 199-205.

\title{
A Historical Note: An anesthetic disaster
}

As a first year resident in a Toronto teaching hospital during a dry, cold January day in 1965, I became involved in an anesthetic disaster. The senior anesthesiologist was manually ventilating a patient having a hysterectomy using cyclopropane, which was highly explosive, for maintenance. Every effort was made to reduce electrical leakage and "static build-up" by avoiding cautery and electronic monitoring.

However, on that cold, dry day, a static charge was possibly created in the operating room (OR) in association with a leak in the anesthetic circuit. The result was a massive explosion in the OR which blew up the gas machine, created a blast injury to the patient's lungs and left the OR and its staff in complete confusion and disarray. As the resident on call, I rushed to the OR and was able to get another gas machine and ventilate the patient's lungs with $\mathrm{O}_{2}$.

The OR was littered with broken glass, fragments of rubber tubing, and bits of metal, but no gas cylinders exploded. The OR staff had transient deafness, but were able to rapidly complete the surgical procedure. The patient continued to have vital signs, but was bleeding from the endotracheal tube as a result of the blast injury. I continued manual ventilation with $\mathrm{O}_{2}$ as there was no mechanical ventilator available.
I transferred the patient to the intensive care unit (ICU) and continued ventilation with the aid of a Bird Mark VII pressure controlled ventilator. As the resident also on call for the ICU, I watched the patient deteriorate due to pulmonary hemorrhage and lack of lung tissue for gas exchange. There was no ECMO at that time and lung transplant had not even been thought of.

Historical incidents such as the one that I was involved in, should make us thankful that one of the great dangers to patients and OR personnel is now a thing of the past in modern anesthesia. The use of explosive agents such as ether and cyclopropane is no longer even considered due to the array of electrical equipment in the OR. Cyclopropane was a wonderful agent, but it explodes! A generation of anesthesiologists who began practice after 1965 will never see it used in Canada.

Gordon Sellery

London, Ontario 